\title{
Testing Distinct Three-Dimensional Effects in Laparoscopy: A Prospective Randomized Trial Using the Lübecker Toolbox Curriculum
}

\author{
Michael Thomaschewski ${ }^{a}$ Thorsten Jürgens $^{\mathrm{b}}$ Claudia Benecke $^{\mathrm{a}}$ \\ Anna-Catherina Griesmann ${ }^{a}$ Hamed Esnaashari ${ }^{a}, c$ Romy Lux ${ }^{a}$ \\ Diana Scheppan $^{\mathrm{a}}$ Ronja Simon ${ }^{\mathrm{a}}$ Tobias Keck $^{\mathrm{a}}$ Tilman Laubert ${ }^{\mathrm{a}, \mathrm{c}}$ \\ ${ }^{a}$ Department of Surgery, University Medical Center Schleswig-Holstein, Campus Lübeck, Lübeck, Germany; \\ b Olympus Surgical Technologies Europe, Hamburg, Germany; ' LTB Germany Ltd., Lübeck, Germany
}

\section{Keywords}

3D laparoscopy · Imaging · Laparoscopic surgery · Stereo base $\cdot$ Surgical training

\begin{abstract}
Background: The use of stereoscopic laparoscopic systems in minimally invasive surgery (MIS) allows a three-dimensional (3D) view of the surgical field, which improves handeye coordination. Depending on the stereo base used in the construction of the endoscopes, 3D systems may differ regarding the $3 \mathrm{D}$ effect. Our aim was to investigate the influence of different stereo bases on the 3D effect. Methods: This was a prospective randomized study involving 42 MISinexperienced study participants. We evaluated two laparoscopic 3D systems with stereo bases of $2.5 \mathrm{~mm}$ (system A) and $3.8 \mathrm{~mm}$ (system B) for differences in learning MIS skills using the Lübeck Toolbox (LTB) video box trainer. We evaluated participants' performance regarding the times and repetitions required to reach each exercise's goal. After completing the final exercise ("suturing"), participants performed the exercise again using a two-dimensional (2D) representation. Additionally, we retrospectively compared our study results with a preliminary study from participants completing the LTB curriculum with a 2D system. Results: The median number of repetitions until reaching the goals for LTB exercises 1, 2, 3, and 6 for system A were: 18 (range 7-53), 24 (range 8-46), 24 (range 13-51), and 21 (range 10-46), respectively, and for system B were: 12 (range 2-30), 16 (range
\end{abstract}

6-43), 17 (range 4-47), and 15 (range 6-29), respectively ( $p=$ not significant). Changing from a 3D to a $2 \mathrm{D}$ representation after completing the learning curve led to a longer average time required, from 95.22 to $119.3 \mathrm{~s}(p<0.0001)$, for the last exercise (exercise 6; "suturing"). When comparing the results retrospectively with the learning curves acquired with the $2 \mathrm{D}$ system, there was a significant reduction in the number of repetitions required to reach the LTB exercise goals for exercises 1, 3, and 6 using the 3D system. Conclusion: Stereo bases of 2.5 and $3.8 \mathrm{~mm}$ provide acceptable bases for designing 3D systems. Additionally, our results indicated that MIS basic skills can be learned quicker using a 3D system versus a 2D system, and that when the $3 \mathrm{D}$ effect is eliminated, the corresponding compensatory mechanisms must be relearned.

(c) 2020 S. Karger AG, Basel

\section{Introduction}

Learning minimally invasive surgery (MIS) procedures is highly challenging for surgeons and results in flatter learning curves compared with the corresponding open procedures [1]. Compared with open surgery, MIS requires additional psychomotor skills such as different haptic competencies, and opposing movements must be applied to the instruments fixed at the level of the body wall (fulcrum effect) [1]. However, the cognitive transfer of a two-dimensional (2D) monitor image to the three- 
dimensional (3D) surgical field is most challenging. With a $2 \mathrm{D}$ representation, the brain can no longer determine depth information via stereopsis or through the oculomotor system, and corresponding compensatory mechanisms must be learned $[2,3]$.

To avoid the problem of the missing depth perception, 3D laparoscopic systems have been introduced in MIS. The depth perception thus achieved should lead to improved hand-eye coordination and easier learning, and performing MIS procedures with shorter operation times [3-7].

When constructing a laparoscopic 3D optic, the distance between the left and right optical channels (stereo base) and the orientation of the two optical channels to each other (stereoscopic angle) must be selected, and determining these parameters represents a compromise. On the one hand, a larger stereo base or a larger stereoscopic angle at the same viewing distance leads to an increased perception of the depth differences presented in the image. On the other hand, realistic stereo base measurements or stereoscopic angles are limited by constructive boundary conditions, and primarily by the desired outer diameter of the endoscope. In addition, excessively large stereo bases and stereoscopic angles can lead to the perception of double images at small working distances and to visual overload for the viewer [8]. However, it remains unclear to what extent different stereo bases and stereoscopic angles influence depth perception and surgical performance during MIS procedures.

To determine the influence of optical parameters on MIS performance, it is most effective to use a standardized MIS simulation tool. Recently, we devised a comprehensive, strictly defined, and goal-directed curriculum to teach MIS basic skills, the Lübeck Toolbox (LTB) curriculum (http://www.luebeck-toolbox.com) $[9,10]$. The LTB curriculum includes a video box-trainer, six iteratively developed tasks, and predefined expert levels for each task, as well as online video tutorials [11] and documentation material for the training progress. The goals for each task were clearly defined based on a benchmark study involving MIS experts followed by a prospective validation study involving MIS novices $[9,10]$. In addition, we previously demonstrated the transferability of the LTB-based skills to an organic surgical model in prospective studies [9]. The extremely high standardization of the exercises in the LTB curriculum enables the system to be used for analyses such as those used in product development of MIS instruments and tools. To investigate the influence of different optical 3D systems on MIS performance, we performed this prospective randomized study comparing the effect of different 3D optics on the learning curves of MIS novices completing the LTB curriculum.

\section{Materials and Methods}

Study Design and Setting

This was a prospective, single-center, two-arm, parallel-group randomized controlled study (Fig. 1). The study was approved by the Ethics Committee of the Lübeck University (protocol No. 16316). This study was performed at the Department of Surgery, University Medical Centre Schleswig-Holstein, Campus Lübeck, Lübeck, Germany.

In addition, this study contained a retrospective analysis in which we compared the learning curves of the present study using $3 \mathrm{D}$ optics with the learning curves using $2 \mathrm{D}$ optics obtained from previous studies.

\section{Objectives}

The primary objective of the study was to compare the effect of different stereo bases on MIS skills acquisition of participants with no prior MIS experience performing the LTB exercises. In the current study, we compared the prototype of a $3 \mathrm{D}$ video laparoscope with a stereo base of $2.5 \mathrm{~mm}$ and a stereoscopic angle of $3.58^{\circ}$ (EN-

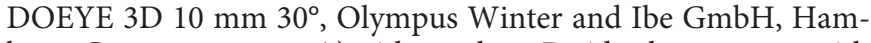
burg, Germany; system A) with another 3D video laparoscope with a stereo base of $3.8 \mathrm{~mm}$ and a stereoscopic angle of 5.44 (LTF-19010-3D, Olympus Medical Systems Corp., Tokyo, Japan; system B; Table 1). After randomization into two groups, participants performed the LTB exercises with either system A or system B (Fig. 1). After completing the LTB exercises, we compared the study groups with regard to two aspects addressing the effect of the different stereo bases as follows: (i) we compared the learning curves of both groups and recorded which group reached the objectives of the LTB exercises faster, i.e., with fewer task repetitions, and (ii) using a questionnaire that participants completed after each exercise, we determined and compared the extent of participants' subjective perceptions of visual overload in both groups.

Additionally, to examine the transferability of the MIS skills acquired using the 3D laparoscopic systems to 2D laparoscopic systems, all study participants performed five repetitions of the last LTB exercise 6 ("suturing") using a 2D system. The number of required repetitions for the successful completion of the exercise with a $2 \mathrm{D}$ system were then determined and compared between the groups. In an additional analysis, the learning curves of the MIS skills achieved using the 3D optics systems were retrospectively compared with learning curves using 2D optics obtained from earlier studies [9].

\section{Study Participants}

Medical students from the third year onwards were recruited as participants. It was ensured that all participants were able to perform a surgical suture in open technique. This prerequisite was necessary to avoid affecting the learning curves of the suturing exercise by having to learn the basic principle of a surgical suture and knot. At the beginning of the study, participants were not permitted to have performed any prior MIS simulation training or to have had any previous MIS exposure in the operating room. To avoid affecting the study results because of insufficient visual acuity or missing stereopsis, all study participants had to demonstrate visual acuity of at least 1.0 and have normal stereopsis which was tested using a Lang Stereo Test (LANG STEREOTEST I, Hübel, Germany). All participants provided their informed consent.

\section{Randomization}

Randomization was performed with stratification regarding gender and dominant hand as permutations of blocks of different lengths with a 1:1 ratio. The study participants were then informed of which group she or he was assigned to (group A used system A, and group B used system B). 


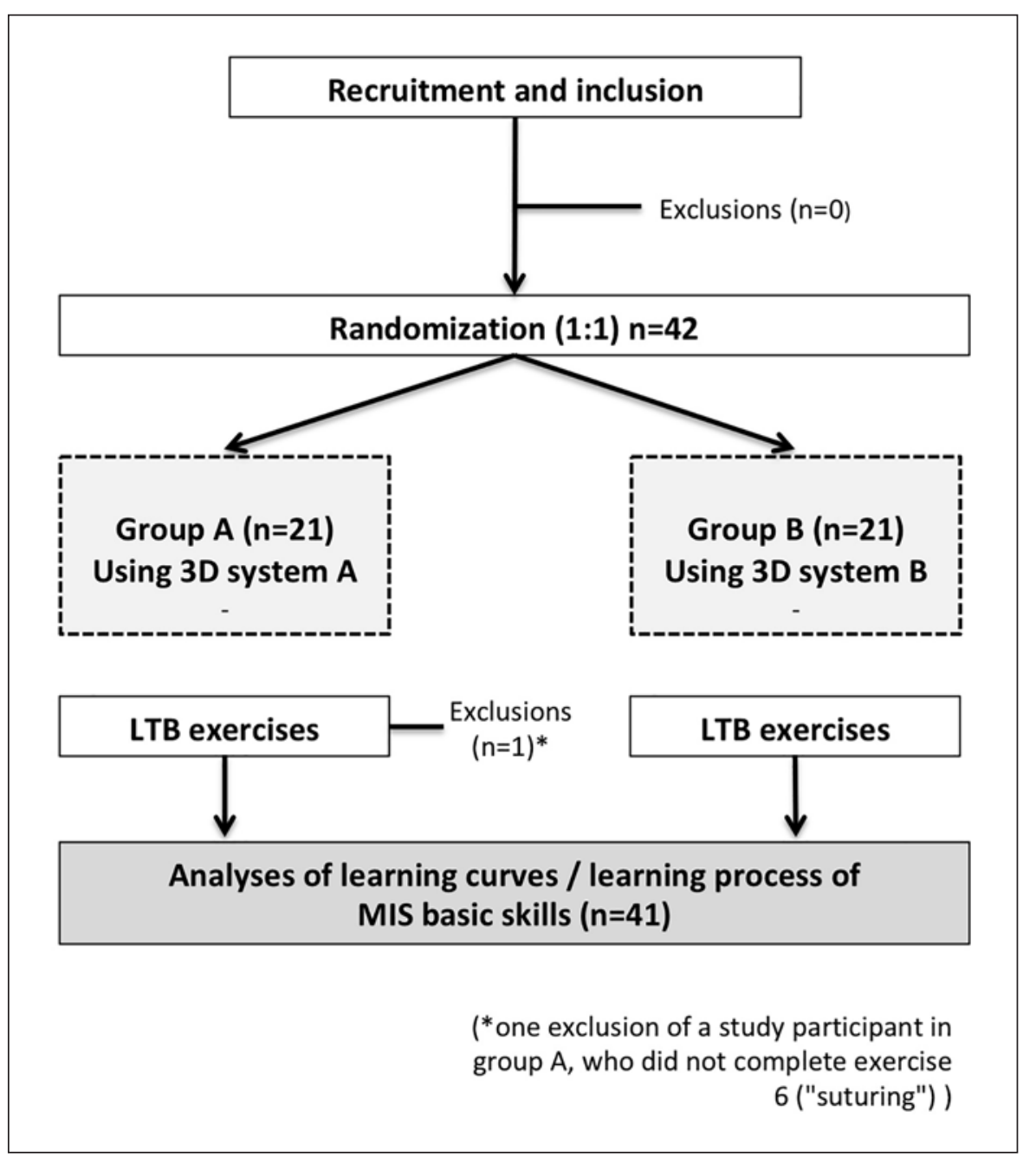

Fig. 1. Study design.

Table 1. The properties of the optics (system A and B) compared in this study

\begin{tabular}{llll}
\hline & & $\begin{array}{l}\text { Stereo } \\
\text { base, mm }\end{array}$ & $\begin{array}{l}\text { Stereoscopic } \\
\text { angle }{ }^{\mathrm{a}}{ }^{\circ}\end{array}$ \\
\hline System A & ENDOEYE 3D 10 mm 30 & 2.5 & 3.58 \\
System B & LTF-190-10-3D & 3.8 & 5.44 \\
\hline
\end{tabular}

${ }^{a}$ At a $40-\mathrm{mm}$ viewing distance.

\section{Primary Outcome Measure}

The primary outcome measure was the learning speed of the MIS basic skills, which was determined by the number of repetitions necessary to achieve the goals of the LTB exercises with the predefined precision.

\section{Secondary Endpoints}

As a secondary endpoint, we determined the number of participants who were unable to successfully complete the LTB exercises. We also determined and compared the extent of participants' subjective perception of visual overload while performing the LTB exercises in both groups using a questionnaire with the following six items: fatigue (Q1), dry eyes (Q2), eye strain (Q3), accommodation difficulties (Q4), double vision (Q5), and headaches (Q6). The questionnaire was completed after each training session. This questionnaire was based on a study on visual fatigue at computer workstations [12]. The transferability of the MIS skills acquired by the study participants to a $2 \mathrm{D}$ system was determined by the average exercise time of five repetitions of task 6 ("suturing") and compared with the exercise times of the $3 \mathrm{D}$ systems, between the groups.

\section{Retrospective Data from a Preliminary Study Using a 2D}

System

In a retrospective comparative design, the learning outcomes of MIS training with the 3D systems (A and B) were compared with the data of a preliminary study performed with a 2D system [9]. The study design of the preliminary study, in which the LTB exercises were performed with a 2D optic, was entirely analogous to the protocol used in the present study. The only difference was that participants in the preliminary study had completed the entire LTB curriculum, including exercises 4 and 5.

\section{Statistics}

All statistical test procedures and diagrams were generated using GraphPad Prism statistical software (GraphPad Prism version 6.07 for Windows, GraphPad Software, San Diego, CA, USA; www.graphpad.com). 
A

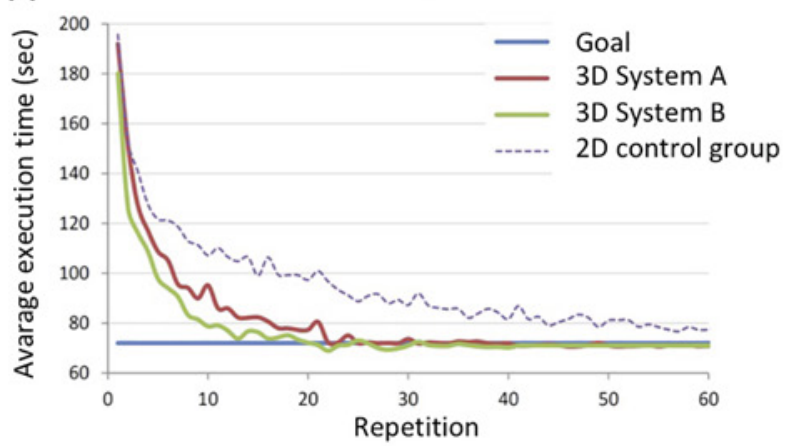

C

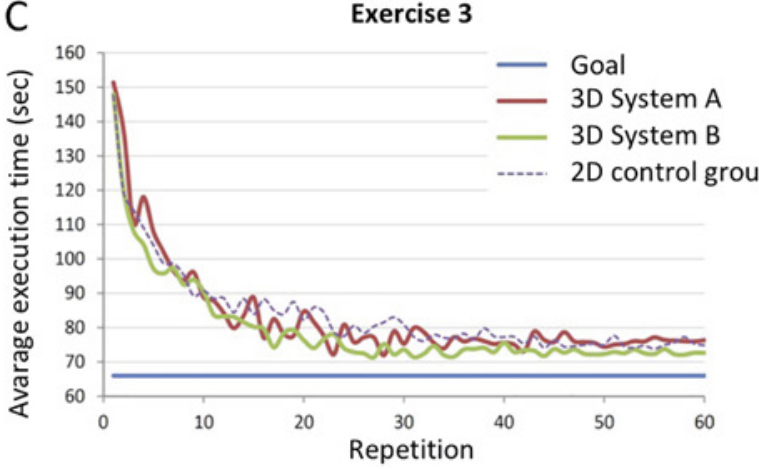

B $\quad$ Exercise 2

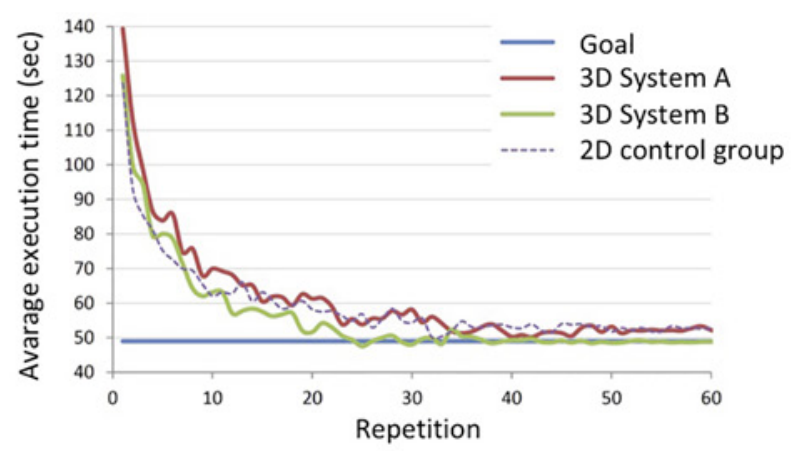

D

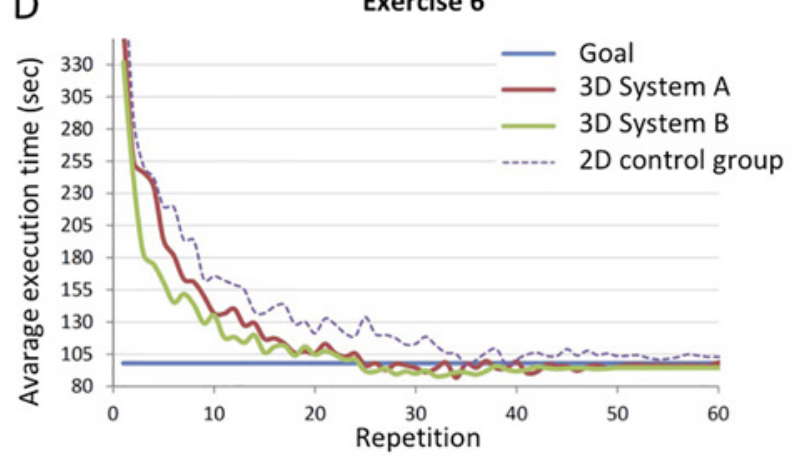

Fig. 2. Learning curves of LTB exercises 1 (A), 2 (B), 3 (C), and 6 (D).

Using the D'Agostino-Pearson test, we evaluated whether the data were normally distributed. We set the probability of error at $\alpha=5 \%$. To determine the influence of the different systems on learning speed, we determined the relative effect size using the mean values of the required repetitions of the LTB exercises.

For simple comparisons, we used a two-sample $t$ test for normally distributed data; otherwise we used Wilcoxon's rank sum test. For multiple comparisons, we used Tukey's test on normally distributed data, and the Kruskal-Wallis test followed by Dunn's post test, in the absence of a normal distribution. We used contingency tables to evaluate the completed questionnaires to determine visual overload. $p$ values were calculated using Fisher's exact test, and for all analyses $p<0.05$ was considered statistically significant. In addition, we generated box plots for a graphical evaluation, which, in addition to the individually achieved characteristic values for each participant, showed the median as well as 25 (lower quartile) and 75 percentiles (upper quartile) of the respective data series.

\section{LTB Training Exercises}

For this study, exercises No. 1 ("pack your luggage”), No. 2 ("weaving"), No. 3 ("Chinese jump rope"), and No. 6 ("suturing") of the LTB curriculum were used for the MIS training program and were performed as described previously $[9,10]$. We did not use exercises No. 4 ("triangle cut") and No. 5 ("hammer cut") of the LTB curriculum under the assumption that the selected exercises required a higher spatial perception than the excluded exercises, so that possible differences between the $3 \mathrm{D}$ systems would result in greater effects. After completing the last exercise in $3 \mathrm{D}$ mode, all participants performed five repetitions of the last exercise (exercise No. 6 "suturing") in 2D mode. One image sensor of each 3D system was deactivated so that the monitors output a $2 \mathrm{D}$ image.

\section{Materials and Equipment}

The properties of the optics compared in this study are shown in Table 1 (system A and B). The laparoscopic images for both system $A$ and system $B$ were displayed using a video system consisting of two Evis Exera CV 190 video processors (Olympus Medical Systems Corp.), a 3DV-190 video mixer (Olympus Medical Systems Corp.), and an Evis Exera CLV 190 laparoscopic light source (Olympus Medical Systems Corp.). The 3D video image was displayed on a 3D monitor, and participants wore polarized glasses (LMD 2451 MT, Sony Corp., Tokyo, Japan). The viewing distance between the study participant and the monitor was $80 \pm 20 \mathrm{~cm}$, and the training module was positioned on the optical axis between the study participant and the monitor.

The LTB video box trainer was used for the MIS training (LTB Germany Ltd., Lübeck, Germany). Originally, the LTB system was equipped with its own video camera and did not provide access for a laparoscope. Therefore, we modified the boxtrainer by placing a holder on the box, in which the endoscopes could be aligned and fixed. The instruments used for the LTB exercises were atraumatic grasping forceps (Endopath 5-mm Overholt 5DCD; Ethicon EndoSurgery, Cincinnati, OH, USA) and needle drivers (Endopath 5-mm needle holder E705R; Ethicon Endo-Surgery). Vicryl SH PLUS 3-0 (Ethicon, Norderstedt, Germany) was used as suture material. 


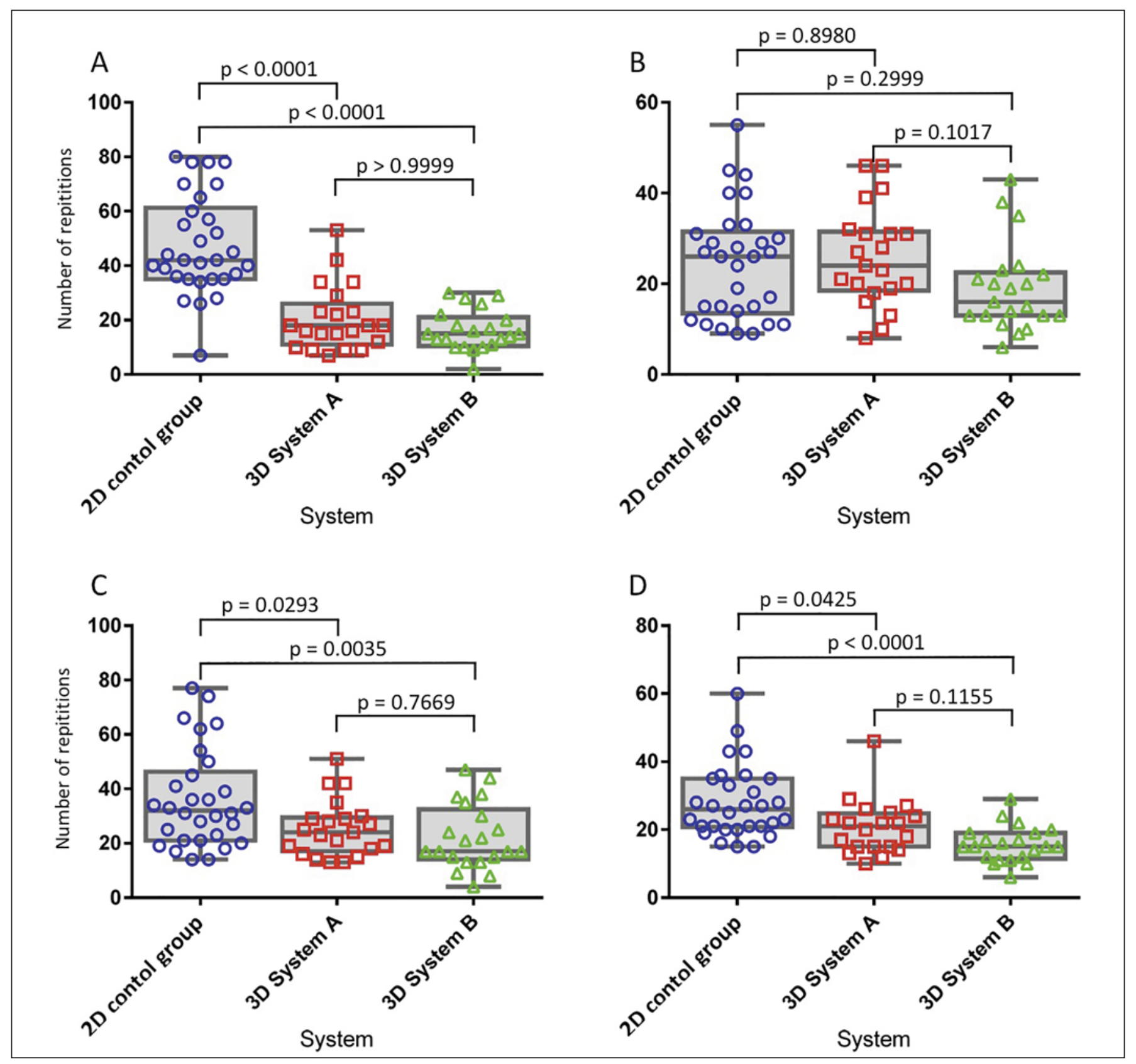

Fig. 3. Required number of repetitions to achieve the goals of LTB exercise 1 (A), 2 (B), 3 (C), and 6 (D).

\section{Results}

Except for one exclusion of a study participant in group A, who did not complete exercise 6 ("suturing"), there were no exclusions during the study period, and 41 out of 42 study participants achieved all required goals of the LTB exercises. No violation of the study protocol occurred.

Comparison of Learning MIS Basic Skills between

System $A$ and $B$

The learning curves of study participants and the required number of repetitions to achieve the goals of LTB exercise No. 1, 2, 3, and 6 are shown in Figures 2 and 3. We found no significant difference between system A and B.

Participants required an average time to complete all LTB exercises of $193.0 \mathrm{~s}$ using system A and $234.1 \mathrm{~s}$ using system B ( $p=$ not significant). The completion times for the individual LTB exercises are shown in Table 2 and Figure 4, and we found no significant difference between system $A$ and $B$.

Influence of System A and B on Participants' Visual Overload

The relative risk of the occurrence of a visual side effect was 1.12 times higher for system B than for system A (Ta- 

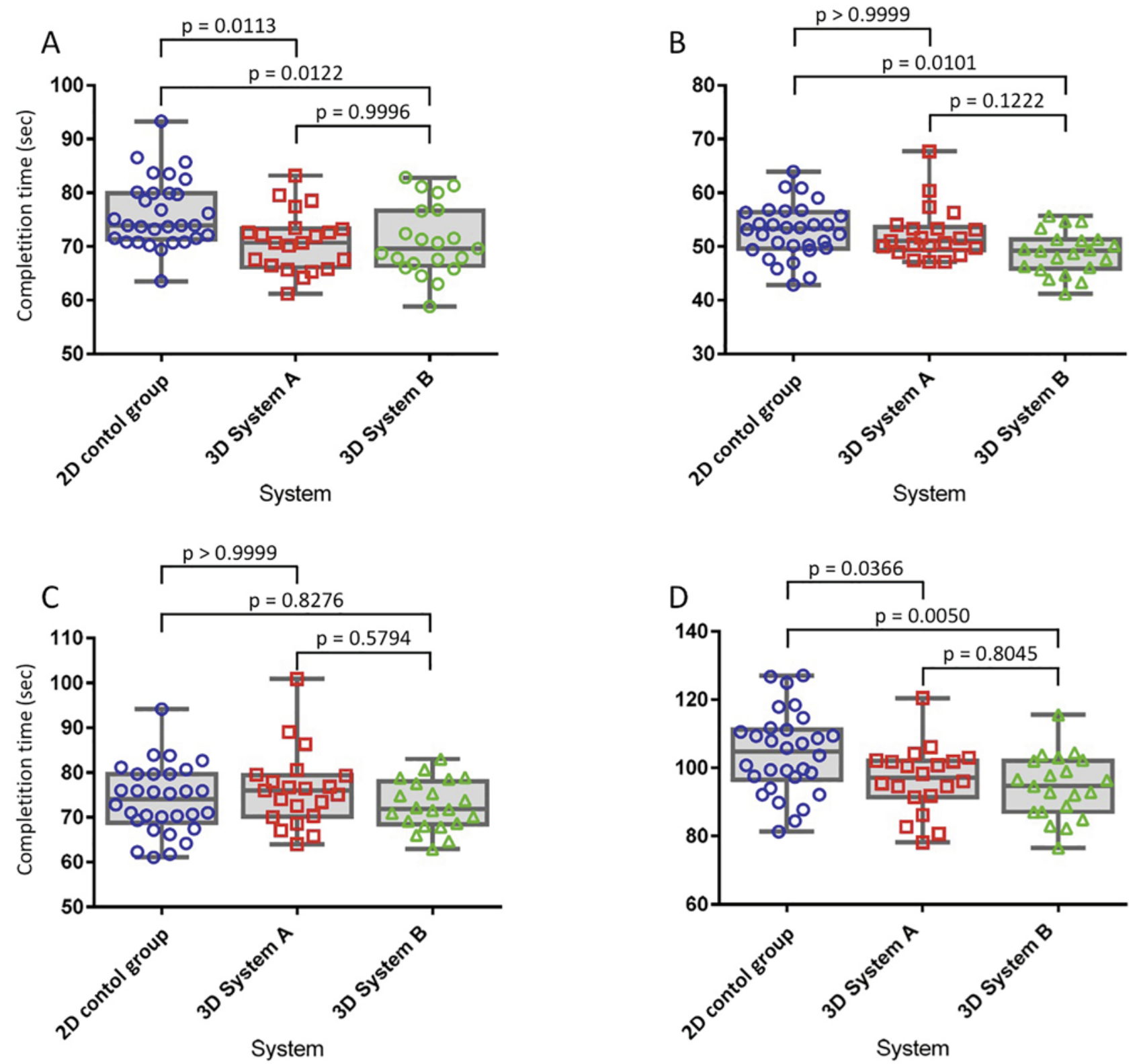

Fig. 4. Completion times for the individual LTB exercises 1 (A), 2 (B), 3 (C), and 6 (D).

Table 2. Completion times for the individual LTB exercises

\begin{tabular}{lccc}
\hline & $\begin{array}{l}\text { 3D system A, } \\
\text { min }\end{array}$ & $\begin{array}{l}\text { 3D system B, } \\
\text { min }\end{array}$ & $\begin{array}{l}\text { 2D control } \\
\text { group, min }\end{array}$ \\
\hline Exercise 1 & 47.2 & 38.1 & 91.7 \\
Exercise 2 & 45.0 & 35.2 & 41.2 \\
Exercise 3 & 52.3 & 47.1 & 66.7 \\
Exercise 6 & 89.5 & 72.7 & 113.6 \\
\hline Total & 234.1 & 193.0 & 313.2 \\
\hline
\end{tabular}

ble 3), but the difference was not statistically significant $(p=0.3408)$. Subsequently, the individual questionnaire contents, which were completed after each training session, were evaluated separately for system A and B. We found no significant difference regarding the occurrence of fatigue (Q1), dry eyes (Q2), eye strain (Q3), accommodation difficulties (Q4), double vision (Q5), and headaches (Q6). However, data for participants using system $\mathrm{B}$ showed a trend towards more frequent occurrence of dry eyes $(\mathrm{Q} 2, p=0.07)$, accommodation difficulties $(\mathrm{Q} 4$, $p=0.06)$, and double vision $(\mathrm{Q} 5, p=0.05)$. 
A

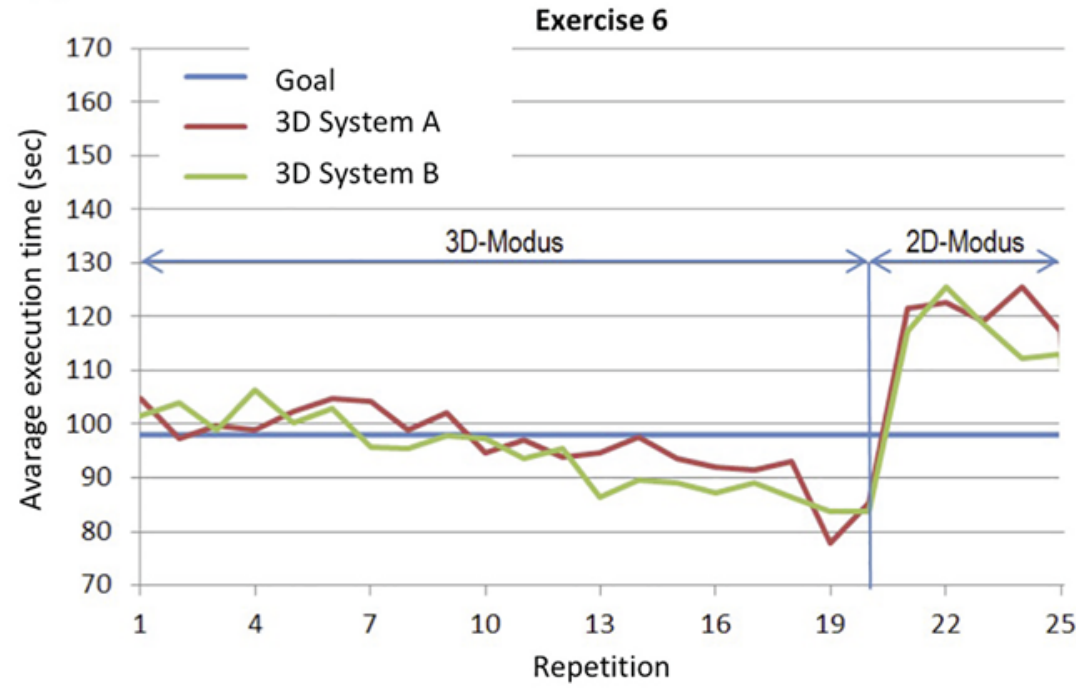

B
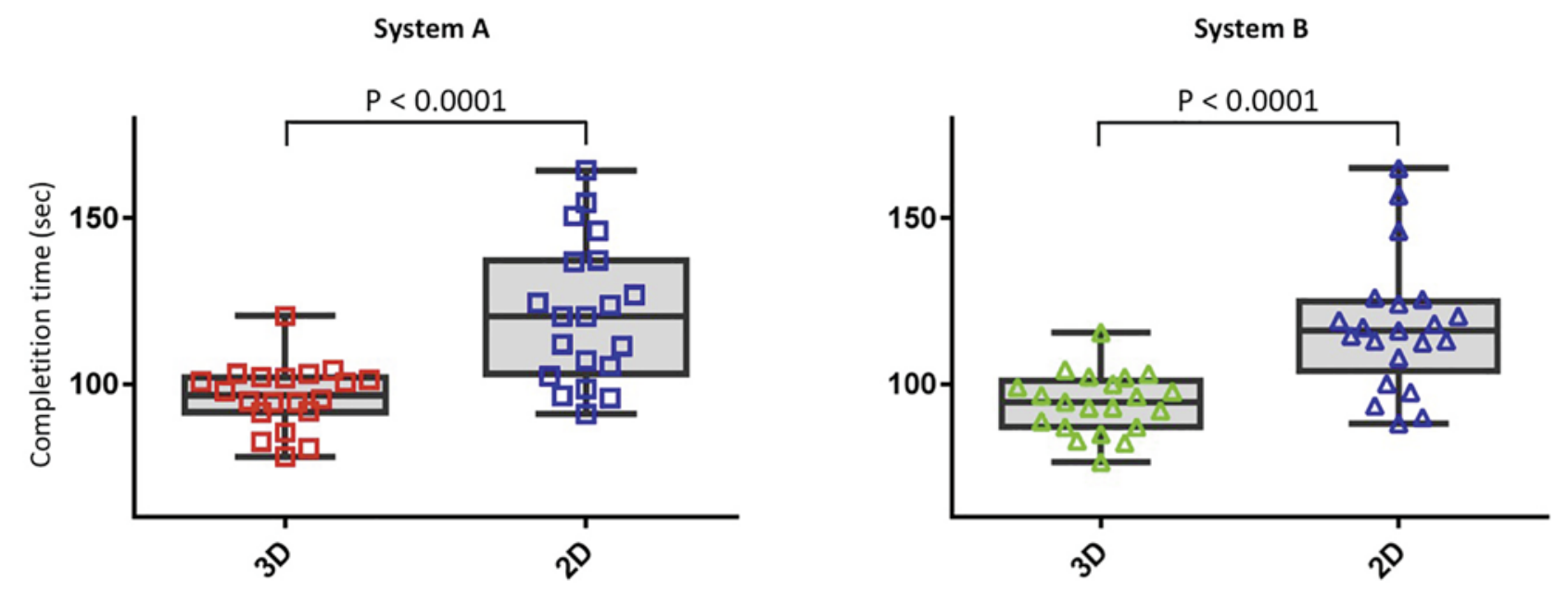

Fig. 5. Transferability of the MIS skills acquired with the 3D systems to a 2D system. A Learning curves of exercise 6 "suturing." B Completion times of exercise 6 "suturing."

Transferability of the MIS Skills Acquired with the $3 D$ Systems to a 2D System

After successfully completing all LTB exercises with the 3D optics (system A and B), participants performed five repetitions of exercise 6 ("suturing") in 2D mode. The average completion time increased from 96.25 to $121.31 \mathrm{~s} \mathrm{in}$ group $\mathrm{A}$, and from 94.25 to $117.43 \mathrm{~s}$ in group $\mathrm{B}$, corresponding to an increase of $26.0 \%$ in group $A$ and $24.6 \%$ in group $\mathrm{B}$. The differences regarding the completion times between the $3 \mathrm{D}$ and the $2 \mathrm{D}$ system were statistically significant $(p<0.0001)$; however, we found no significant difference when comparing systems A and B (Fig. 5).
Comparison of the 3D Data with the Data from a Preliminary Study Using a 2D System

In the preliminary study using the $2 \mathrm{D}$ system, the median number of repetitions needed to achieve the goals of LTB exercises 1, 2, 3, and 6 were as follows: 42 (range $7-80), 26$ (9-55), 32 (14-77), and 26 (15-60), respectively. Compared with the present study using the $3 \mathrm{D}$ systems, participants required significantly fewer repetitions to achieve the goals of LTB exercises $1(p<0.0001$, system $A$ and $B), 3(p=0.029$, system $A ; p=0.004$, system $B)$, and 6 ( $p=0.043$, system A; $p<0.0001$, system B). In contrast, the required number of repetitions for exercise 2 showed 
Table 3. Occurrence of visual side effects determined via a questionnaire after each training session

\begin{tabular}{|c|c|c|c|c|}
\hline & & $\begin{array}{l}\text { 3D system } \\
\text { A, \% }\end{array}$ & $\begin{array}{l}\text { 3D system } \\
\text { B, \% }\end{array}$ & $p$ value \\
\hline (Q1) Fatigued eyes & yes & 13.33 & 14.78 & 0.2350 \\
\hline (Q2) Dry eyes & yes & 13.33 & 7.56 & 0.0676 \\
\hline (Q3) Eye strain & yes & 2.12 & 2.06 & 1 \\
\hline (Q4) Accommodation & & & & \\
\hline difficulties & yes & 7.88 & 12.71 & 0.0616 \\
\hline (Q5) Double vision & yes & 0.61 & 2.75 & 0.0517 \\
\hline (Q6) Headaches & yes & 1.82 & 1.72 & 1 \\
\hline Total (Q1-Q6) & yes & 6.21 & 6.93 & 0.4135 \\
\hline
\end{tabular}

no significant differences compared with the study using $2 \mathrm{D}$ optics $(p=0.898$, system $\mathrm{A} ; p=0.3$, system $\mathrm{B}$; Fig. 2, 3).

When considering the total time required to achieve the goals of all of the LTB exercises, participants were faster with the 3D systems compared with the comparative study using the $2 \mathrm{D}$ system (mean value for system A 234.1 vs. $193.0 \mathrm{~min}$ for system B vs. $313.2 \mathrm{~min}$ for the 2D study; Table 2). Considering individual LTB exercises, the participants' average execution times required in the 3D groups were significantly shorter compared with the 2D cohort for LTB exercise 1 ( $p=0.011$, system $\mathrm{A} ; p=0.012$, system $\mathrm{B})$ and exercise $6(p=0.037$, system A; $p=0.005$, system B; Fig. 4). Comparative results for exercise 3 showed no significant difference $(p>0.999$, system $A ; p=0.828$, system B; Fig. 4 ). For exercise 2 , we found significantly shorter times required regarding system B $(p=0.010)$, whereas system A showed no significant difference compared with the $2 \mathrm{D}$ group $(p>$ 0.999; Fig. 4).

\section{Discussion}

Learning laparoscopically assisted surgical procedures is more complex and demanding than for the corresponding open procedures, especially for complex procedures. Most importantly, the lack of depth perception through the $2 \mathrm{D}$ representation of the surgical field requires additional learning and the use of cognitive compensatory mechanisms [1-3]. To address this difficulty, laparoscopic 3D systems may facilitate learning and implementing MIS procedures [3-7]. However, it is unclear to what extent different stereo bases and stereoscopic angles - and, consequently, the intensity of a 3D depiction - have an influence on depth perception and operative performance. This question plays an important role in the construction of laparoscopic optics, as a small diameter is needed to minimize surgical trauma; however, smaller diameters limit the width of the stereo base and the stereoscopic angle and, thus, the depth perception.

In the present study, we compared two laparoscopic 3D systems with different stereo bases and stereoscopic angles. In this prospective randomized study, we compared the learning speed of MIS basic skills using the LTB video box MIS trainer, which consists of highly standardized exercise modules and procedures.

Our results indicated that a larger stereo base positively enhanced depth perception. For each LTB exercise, group $B$ participants were able to reach the goal earlier on average than participants in group $\mathrm{A}$, who used the smaller stereo base system; however, the differences were not statistically significant. Based on our results, we concluded that system A and system B are both acceptable for 3D system design. To our knowledge, ours is the first study investigating the effect of different stereo bases and stereoscopic angles on MIS learning performance.

No significant differences were found regarding whether the stereo base and stereoscopic angle influenced the occurrence of visual overload effects, although there was a nearly significant difference in the frequency of dry eyes, accommodation difficulties, and double vision using the larger stereo base (system B). However, we did not have comparative data for the $2 \mathrm{D}$ optics system. In studies by Tanagho et al. [7] and Alaraimi et al. [4] investigating visual overload effects such as eye strain, headaches, vertigo, orientation difficulties, and discomfort, no significant difference was found between $2 \mathrm{D}$ and 3D laparoscopic systems. In contrast, some studies reported less strain using $3 \mathrm{D}$ versus $2 \mathrm{D}$ systems [13]. It should also be considered that the training exercises and thus the $3 \mathrm{D}$ exposure, i.e., duration task repetition and duration of training session, both in our study and in the studies by Tanagho et al. [7] and Almari et al. [4] were possibly too short for the occurrence of effects of visual overload. However, this may be different for prolonged 3D exposure, for example during long surgical procedures. In summary, we can make no clear statement regarding whether the size of the stereo base and the stereoscopic angles that we evaluated have an influence on the occurrence of visual overload effects; however, our results indicated a comparable effect.

In our retrospective comparative analysis, we compared our data obtained using the $3 \mathrm{D}$ systems with data previously collected with a $2 \mathrm{D}$ system to determine the usefulness of $3 \mathrm{D}$ visualization on the acquisition of MIS basic skills. The extremely high standardization of the LTB contents and the equity of the study protocols regarding training duration and frequency as well as the inclusion criteria for the study participants enabled a retrospective comparison of the different study cohorts [9]. The retrospective comparison of both study co- 
horts showed that using a 3D system had a measurable and positive influence on the learning speed of MIS basic skills. One explanation could be that 3D users do not need to learn mechanisms to compensate for the lack of depth perception in a $2 \mathrm{D}$ visualization. This hypothesis is supported by the fact that the study participants in the $2 \mathrm{D}$ group required significantly longer exercise times during the first exercise to reach the exercise goals, and when performing further exercises, the differences decreased or disappeared. Thus, the advantage of using a 3D system during MIS training appeared to decrease.

Interestingly, the dispersion in the number of required repetitions to reach the goal of LTB exercise 1 was greater in the $2 \mathrm{D}$ group compared with the $3 \mathrm{D}$ group, and in subsequent exercises the dispersion decreased in the $2 \mathrm{D}$ group. Possibly, participants were differently talented regarding the acquisition of compensatory mechanisms for the lack of depth perception. However, after learning the compensatory mechanisms, talent or predispositions no longer appeared to play a relevant role. However, further studies are required to examine this effect more closely.

The fact that additional compensatory mechanisms have to be learned when depth perception is eliminated by $2 \mathrm{D}$ representation was confirmed by our results. After completion of all the LTB exercises in 3D mode, participants required more repetitions of the last exercise (exercise 6; "suturing") in 2D mode. The loss of 3D visualization led to a significant increase in the time needed to complete the task; however, because the skills required for the exercise regarding using the MIC instruments had already been acquired, deactivating the $3 \mathrm{D}$ mode led to a decline in MIS performance, but not to a return to the beginning of the learning curve. In summary, the statistically significant differences in achieving the LTB exercise goals between the $3 \mathrm{D}$ systems and the $2 \mathrm{D}$ system and the lack of significant differences among the stereo bases support the fact that the investigated stereo bases are acceptable options when designing stereoscopic optics for use in MIS.

In accordance with our results, overall published data on the learning effect of MIS using $2 \mathrm{D}$ versus $3 \mathrm{D}$ imaging shows that 3D imaging leads to improved hand-eye coordination and shorter learning curves [4-7, 14]. Even more, prospective randomized trials showed that MIS operation times in the operation room can be shortened when using 3D video systems $[5,14]$. This led to the recently published recommendation of the European Association for Endoscopic Surgery (EAES) consensus development conference 2018 to use 3D laparoscopy to decrease operation time [15].

Our results showed that study participants using the 3D laparoscopic systems showed significantly poorer MIS performance when changing to $2 \mathrm{D}$ mode. Conse- quently, 3D imaging in MIS training decreases acquisition of an MIS basic skill, namely dealing with the lack of depth perception, when 2D visualization is used [16].

With today's use of robotic-assisted surgery, 3D imaging has gained substantial relevance. The most commonly used system, the daVinci ${ }^{\circledR}$ Surgical System (Intuitive Surgical, Sunnyvale, CA, USA), is equipped with a highdefinition $3 \mathrm{D}$ optic to compensate for the complete loss of haptics, among other factors [17]. Regarding the further development of robotic-assisted surgery, our investigation of the effect of different stereo bases on depth perception could also become relevant.

\section{Limitations}

The following are possible limitations of this study. First, it is unclear whether our results are transferable to actual MIS procedures in the operation room. However, there are indications that a surgeon's MIS performance using video box trainers or simulators is transferable to the operation room [18]. Regarding the LTB system used in the current study, the transferability of MIS performance was demonstrated by a laparoscopic cholecystectomy in a porcine model [9]. Another limitation is that we evaluated the effect of the optical parameters with participants who were novices in MIS. Whether the results are transferable to more experienced surgeons remains unclear and must be investigated in further studies. Third, the results of our comparison analysis between the $2 \mathrm{D}$ and $3 \mathrm{D}$ systems should be viewed with caution, because the data were collected at different times from distinct studies. Finally, prospective studies involving an industrial partner more often report positive results compared with non-industry-funded trials [19]. The fact that our study was funded by Olympus Surgical Technologies Europe, and two of the coauthors (H.E. and T.L.) are cofounders of LTB Ltd. may have influenced the interpretation and presentation of the results.

\section{Conclusion}

Our comparison of the 3D systems used in this study showed a tendency that the larger of the two stereo bases ( 2.5 vs. $3.8 \mathrm{~mm}$ ), with a related larger 3D effect, led to an improvement in learning MIS basic skills, but at the same time could promote visual overload. However, the differences were not statistically significant, suggesting that the sizes used in system A and system B are both acceptable when designing 3D systems. In addition, MIS basic skills may be learned quicker with a $3 \mathrm{D}$ system than with a $2 \mathrm{D}$ system; however, once the 3D effect is eliminated, the corresponding compensatory mechanisms must be relearned when changing from a $3 \mathrm{D}$ to a $2 \mathrm{D}$ system. 


\section{Statement of Ethics}

This protocol study received approval from the Ethics Committee of the University of Lübeck (protocol No. 16-316). Written informed consent was obtained from those who agreed to participate.

\section{Disclosure Statement}

This study was funded by Olympus Surgical Technologies Europe. Aside from that fact, Michael Thomaschewski, Claudia Benecke, Anna-Catherina Griesmann, Romy Lux, Diana Scheppan, Ronja Simon, and Tobias Keck have no conflicts of interest and/or financial ties to disclose.

Tilman Laubert and Hamed Esnaashari are cofounders of LTB Ltd. Thorsten Jürgens is an employee of Olympus Surgical Technologies Europe. All other authors declare that they have no conflicts of interest.

\section{Funding Sources}

This study was funded by Olympus Surgical Technologies Europe.

\section{Author Contributions}

M.T., T.J., C.B., and T.L. conceived the trial and supplied critical intellectual content. T.J. planned and performed the statistical analysis. C.B., T.K., and H.E. supplied critical intellectual content. A.-C.G., R.L., D.S., and R.S. conducted the study. M.T. and T.L. were major contributors in writing the manuscript. All authors read and approved the final manuscript.

\section{Availability of Data and Materials}

Consent for data sharing cannot be obtained and is not available.

\section{References}

1 Berguer R, Smith WD, Chung YH. Performing laparoscopic surgery is significantly more stressful for the surgeon than open surgery. Surg Endosc. 2001 Oct;15(10):1204-7.

2 Shah J, Buckley D, Frisby J, Darzi A. Depth cue reliance in surgeons and medical students. Surg Endosc. 2003 Sep;17(9):1472-4.

3 Read JC, Begum SF, McDonald A, Trowbridge J. The binocular advantage in visuomotor tasks involving tools. Iperception. 2013 Mar;4(2):101-10.

4 Alaraimi B, El Bakbak W, Sarker S, Makkiyah S, Al-Marzouq A, Goriparthi R, et al. A randomized prospective study comparing acquisition of laparoscopic skills in three-dimensional (3D) vs. two-dimensional (2D) laparoscopy. World J Surg. 2014 Nov;38(11):2746-52.

5 Bilgen K, Ustün M, Karakahya M, Işik S, Sengül S, Cetinkünar S, et al. Comparison of $3 \mathrm{D}$ imaging and 2D imaging for performance time of laparoscopic cholecystectomy. Surg Laparosc Endosc Percutan Tech. 2013 Apr; 23(2):180-3.

6 Roach VA, Mistry MR, Wilson TD. Spatial visualization ability and laparoscopic skills in novice learners: evaluating stereoscopic versus monoscopic visualizations. Anat Sci Educ. 2014 Jul-Aug;7(4):295-301.

7 Tanagho YS, Andriole GL, Paradis AG, Madison KM, Sandhu GS, Varela JE, et al. 2D versus $3 \mathrm{D}$ visualization: impact on laparoscopic proficiency using the fundamentals of laparoscopic surgery skill set. J Laparoendosc Adv Surg Tech A. 2012 Nov;22(9):865-70.

8 Welchman AE. The Human Brain in Depth: How We See in 3D. Annu Rev Vis Sci. 2016 Oct;2(1):345-76.

9 Laubert T, Esnaashari H, Auerswald P, Höfer A, Thomaschewski M, Bruch HP, et al. Conception of the Lübeck Toolbox curriculum for basic minimally invasive surgery skills. Langenbecks Arch Surg. 2018 Mar;403(2):271-8.

10 Laubert T, Thomaschewski M, Auerswald P, Zimmermann M, Brüheim L, Keck T, et al. [Implementation of a Laparoscopic Simulation Training in Undergraduate Medical Education - The Lübeck Toolbox-Curriculum]. Zentralbl Chir. 2018 Aug;143(4):412-8.

11 Thomaschewski M, Esnaashari H, Höfer A, Renner L, Benecke C, Zimmermann M, et al. Video tutorials increase precision in minimally invasive surgery training - a prospective randomised trial and follow-up study. Zentralbl Chir. 2019 Apr;144(2):153-62.

12 Saito S, Sotoyama M, Saito S, Taptagaporn S. Physiological indices of visual fatigue due to VDT operation: pupillary reflexes and accommodative responses. Ind Health. 1994; 32(2):57-66.

13 Currò G, La Malfa G, Lazzara S, Caizzone A, Fortugno A, Navarra G. Three-Dimensional Versus Two-Dimensional Laparoscopic Cho- lecystectomy: Is Surgeon Experience Relevant? J Laparoendosc Adv Surg Tech A. 2015 Jul;25(7):566-70.

14 Sørensen SM, Savran MM, Konge L, Bjerrum F. Three-dimensional versus two-dimensional vision in laparoscopy: a systematic review. Surg Endosc. 2016 Jan;30(1):11-23.

15 Arezzo A, Vettoretto N, Francis NK, Bonino MA, Curtis NJ, Amparore D, et al. The use of 3D laparoscopic imaging systems in surgery: EAES consensus development conference 2018. Surg Endosc. 2019 Oct;33(10):3251-74.

16 Mistry M, Roach VA, Wilson TD. Application of stereoscopic visualization on surgical skill acquisition in novices. J Surg Educ. 2013 Sep-Oct;70(5):563-70.

17 Sinha RY, Raje SR, Rao GA. Three-dimensional laparoscopy: principles and practice. J Minim Access Surg. 2017 Jul-Sep;13(3):1659.

18 Sroka G, Feldman LS, Vassiliou MC, Kaneva PA, Fayez R, Fried GM. Fundamentals of laparoscopic surgery simulator training to proficiency improves laparoscopic performance in the operating room-a randomized controlled trial. Am J Surg. 2010 Jan;199(1):115-20.

19 Probst P, Knebel P, Grummich K, Tenckhoff S, Ulrich A, Büchler MW, et al. Industry Bias in Randomized Controlled Trials in General and Abdominal Surgery: An Empirical Study. Ann Surg. 2016 Jul;264(1):87-92. 